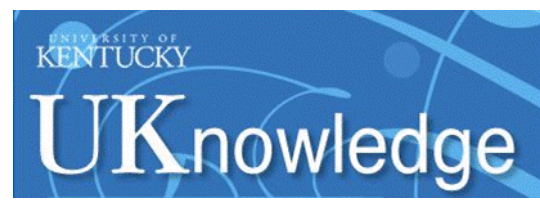

University of Kentucky

UKnowledge

4-17-2013

\title{
Suppression of Dielectronic Recombination due to Finite Density Effects
}

D. Nikolić

Western Michigan University

T. W. Gorczyca

Western Michigan University

K. T. Korista

Western Michigan University

Gary J. Ferland

University of Kentucky, gary@uky.edu

N. R. Badnell

University of Strathclyde, UK

Follow this and additional works at: https://uknowledge.uky.edu/physastron_facpub

Part of the Astrophysics and Astronomy Commons, and the Physics Commons

Right click to open a feedback form in a new tab to let us know how this document benefits you.

\section{Repository Citation}

Nikolić, D.; Gorczyca, T. W.; Korista, K. T.; Ferland, Gary J.; and Badnell, N. R., "Suppression of Dielectronic Recombination due to Finite Density Effects" (2013). Physics and Astronomy Faculty Publications. 61. https://uknowledge.uky.edu/physastron_facpub/61

This Article is brought to you for free and open access by the Physics and Astronomy at UKnowledge. It has been accepted for inclusion in Physics and Astronomy Faculty Publications by an authorized administrator of UKnowledge. For more information, please contact UKnowledge@lsv.uky.edu. 


\section{Suppression of Dielectronic Recombination due to Finite Density Effects}

\section{Digital Object Identifier (DOI)}

https://doi.org/10.1088/0004-637X/768/1/82

\section{Notes/Citation Information}

Published in The Astrophysical Journal, v. 768, no. 1, 82, p. 1-9.

(C) 2013. The American Astronomical Society. All rights reserved. Printed in the U.S.A.

The copyright holder has granted permission for posting the article here. 


\title{
SUPPRESSION OF DIELECTRONIC RECOMBINATION DUE TO FINITE DENSITY EFFECTS
}

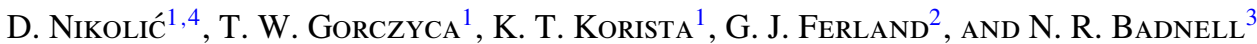 \\ ${ }^{1}$ Western Michigan University, Kalamazoo, MI, USA \\ ${ }^{2}$ University of Kentucky, Lexington, KY, USA \\ ${ }^{3}$ University of Strathclyde, Glasgow, UK \\ Received 2012 October 27; accepted 2013 March 8; published 2013 April 17
}

\begin{abstract}
We have developed a general model for determining density-dependent effective dielectronic recombination (DR) rate coefficients in order to explore finite-density effects on the ionization balance of plasmas. Our model consists of multiplying by a suppression factor those highly-accurate total zero-density DR rate coefficients which have been produced from state-of-the-art theoretical calculations and which have been benchmarked by experiment. The suppression factor is based upon earlier detailed collision-radiative calculations which were made for a wide range of ions at various densities and temperatures, but used a simplified treatment of DR. A general suppression formula is then developed as a function of isoelectronic sequence, charge, density, and temperature. These densitydependent effective DR rate coefficients are then used in the plasma simulation code Cloudy to compute ionization balance curves for both collisionally ionized and photoionized plasmas at very low $\left(n_{\mathrm{e}}=1 \mathrm{~cm}^{-3}\right)$ and finite $\left(n_{\mathrm{e}}=10^{10} \mathrm{~cm}^{-3}\right)$ densities. We find that the denser case is significantly more ionized due to suppression of DR, warranting further studies of density effects on DR by detailed collisional-radiative calculations which utilize state-of-the-art partial DR rate coefficients. This is expected to impact the predictions of the ionization balance in denser cosmic gases such as those found in nova and supernova shells, accretion disks, and the broad emission line regions in active galactic nuclei.
\end{abstract}

Key words: atomic data - atomic processes - plasmas

Online-only material: color figure

\section{INTRODUCTION}

Astronomical emission or absorption sources have an enormous range of densities. Two examples include the intergalactic medium, with $n_{\mathrm{e}} \sim 10^{-4} \mathrm{~cm}^{-3}$, and the broad emission-line regions of active galactic nuclei, with $n_{\mathrm{e}} \sim 10^{10} \mathrm{~cm}^{-3}$. The gas producing the spectrum is not in thermodynamic equilibrium (Osterbrock \& Ferland 2006), so microphysical processes determine the physical conditions.

The two common cases encountered for ionization are photoionization and collisional (e.g., electron-impact) ionization. In both cases, ions are recombined by dielectronic and radiative recombination, with dielectronic recombination (DR) usually the dominant process for elements heavier than helium. Databases give ionization and recombination rates that are the sum of several contributing processes. Examples include Voronov (1997) for electron impact ionization, Verner \& Yakovlev (1995) for photoionization, and the DR project (Badnell et al. 2003) for DR and Badnell (2006a) for radiative recombination; it is these latter data ${ }^{5}$ which will be of primary interest to us in the present study.

The collisional ionization and recombination rate coefficients used in astrophysics are frequently assumed to depend on temperature but to have no density dependence. The rigorous treatment of density-dependent ionization and recombination rate coefficients is via collisional-radiative modeling. This was introduced by Bates et al. (1962) for radiative recombination only and extended to treat the much more complex case of DR by Burgess \& Summers (1969). Summers applied their

\footnotetext{
4 Also at Department of Mechanical Engineering, University of Alberta, Edmonton, AB, Canada.

5 http://amdpp.phys.strath.ac.uk/tamoc/DATA/
}

techniques to determine density-dependent ionization and recombination rate coefficients, and the consequential ionization balance for collisional plasmas, for H-like thru Ar-like ions. Graphical results were presented for the elements $\mathrm{C}, \mathrm{O}$, and $\mathrm{Ne}$ (Summers 1972) and then N, Mg, and Si (Summers 1974). Reduced temperatures and densities were used so as to enable easy interpolation for other elements. Tables of such recombination rate coefficients were made available only via a Laboratory Report-Summers (1974 \& 1979) — due to their voluminous nature at that point in history. The "difficulty" in utilizing these pioneering data led to some modelers attempting to develop simplified approaches. For example, Jordan (1969) used an approach which was based on truncating the zero-density DR sum over Rydberg states using a simple density-dependent cutoff which itself was based on early collisional-radiative calculations by Burgess \& Summers (1969); a suppression factor was formed from its ratio to the zero-density value and then used more generally. Also, Davidson (1975) simplified the collisional-radiative approach of Burgess \& Summers (1969) and, using hydrogenic atomic data, determined suppression factors for Li-like C IV and O VI. New calculations for CIV were made by Badnell et al. (1993) utilizing more advanced (generalized) collisionalradiative modeling (Summers \& Hooper 1983) and much improved atomic data at collisional plasma temperatures (see the references in Badnell et al. 1993).

All of the above works were for electron collisional plasmas and used rather basic DR data (excluding Badnell et al. 1993) as epitomized in the Burgess (1965) General Formula, viz. a common dipole transition for dielectronic capture, autoionization, and radiative stabilization. The purpose of the present paper is to explore density suppression of DR in photoionized plasmas, and within collisional plasmas, using state-of-the-art DR data which take account of a myriad of pathways not feasible in the early 
works above, but which have been shown to be necessary by comparison with experiment. We wish to gain a broad overview utilizing the large test-suite maintained by the plasma simulation code Cloudy. We utilize an approach to DR suppression which is motivated initially by the detailed collisional-radiative results given in Badnell et al. (1993) for C IV at $T=10^{5} \mathrm{~K}$, along with known scalings to all temperatures, charges, and densities. Using these results as a guideline, a more general suppression formula is then determined by fitting to suppression results from extensive detailed collisional-radiative calculations (Summers 1974 $\&$ 1979) for a wide range of ions at several densities and (high) temperatures. Additional modifications are then introduced to account for low temperature DR.

The outline of the rest of the paper is as follows: in the next section we describe the DR suppression model we use; we then apply this suppression to the zero-density DR data, and use the resultant density-dependent DR data in Cloudy to determine the ionization distribution produced under photoionized and collisional ionization equilibrium at low and moderate densities.

\section{GENERALIZED DENSITY SUPPRESSION MODEL}

We use the following approach, detailed more fully in the subsections below. First, the high-temperature collisionalradiative modeling results of Badnell et al. (1993) for DR suppression in $\mathrm{C}$ IV are parameterized by a pseudo-Voigt profile to study the qualitative behavior of suppression as a function of density and temperature. Next, this formulation is then used as a guideline for developing a more comprehensive suppression formula which is obtained by fitting to collisional radiative data for various isoelectronic sequences, ionic charges, densities, and temperatures (Summers 1974 \& 1979). Lastly, the suppression formulation is extended to low temperatures according to the nature of the sequence-specific DR.

\subsection{High-temperature Suppression for Li-like C IV}

We begin by considering DR of Li-like C IV, for which the density-dependent total DR rate coefficient, and therefore the suppression factor, has been computed rigorously within a collisional-radiative modeling approach (Badnell et al. 1993).

In the electron collisional ionization case, because of the consequential high temperature of peak abundance, DR occurs mainly through energetically high-lying autoionizing states (via dipole core-excitations) for which radiative stabilization is by the core electron into final states just below the ionization limit:

$$
e^{-}+1 s^{2} 2 s \rightarrow 1 s^{2} 2 p n l \rightarrow 1 s^{2} 2 s n l+h v .
$$

In the zero-density limit, the intermediate $1 s^{2} 2 s n l$ states can only decay further via radiative cascading until the $1 s^{2} 2 s^{2}$ final recombined ground state is reached, thereby completing the DR process:

$$
\begin{aligned}
1 s^{2} 2 s n l & \longrightarrow 1 s^{2} 2 s n^{\prime} l^{\prime}+h v_{1} \rightarrow \cdots \\
& \longrightarrow 1 s^{2} 2 s^{2}+h v_{1}+h v_{2}+\cdots
\end{aligned}
$$

For finite electron densities $n_{\mathrm{e}}$, on the other hand, there is also the possibility for reionization via electron impact, either directly or stepwise,

$$
e^{-}+1 s^{2} 2 s n l \longrightarrow 1 s^{2} 2 s n^{\prime} l^{\prime}+e^{-} \rightarrow \cdots \rightarrow 1 s^{2} 2 s+e^{-}+e^{-},
$$

and the probability of the latter pathway is proportional to the electron density $n_{\mathrm{e}}$. Because of this alternative reionization pathway at finite densities, the effective DR rate coefficient $\alpha_{\mathrm{DR}}^{\text {eff }}\left(n_{\mathrm{e}}, T\right)$ is thus suppressed from the zero-density value $\alpha_{\mathrm{DR}}(T)$ by a density-dependent suppression factor $S\left(n_{\mathrm{e}}, T\right)$ :

$$
\alpha_{\mathrm{DR}}^{\mathrm{eff}}\left(n_{\mathrm{e}}, T\right) \equiv S\left(n_{\mathrm{e}}, T\right) \alpha_{\mathrm{DR}}(T) .
$$

From the earlier detailed studies of Davidson (1975) and Badnell et al. (1993), the suppression factor is found to remain unity, corresponding to zero suppression, at lower densities until a certain activation density $n_{\mathrm{e}, \mathrm{a}}$ is reached, beyond which this factor decreases exponentially from unity with increasing density. We have found that this suppression factor, as a function of the dimensionless log density parameter $x=\log _{10} n_{\mathrm{e}}$, can be modeled quite effectively by a pseudo-Voigt profile (Wertheim et al. 1974) — a weighted mixture $\mu$ of Lorentzian and Gaussian profiles of widths $w$ for densities above the activation density $x_{\mathrm{a}}=\log _{10} n_{\mathrm{e}, \mathrm{a}}$ :

$$
\begin{aligned}
& S\left(x ; x_{\mathrm{a}}\right) \\
& \quad= \begin{cases}\mu\left[\frac{1}{1+\left(\frac{x-x_{\mathrm{a}}}{w}\right)^{2}}\right]+(1-\mu)\left[e^{-\left(\frac{x-x_{\mathrm{a}}}{w / \sqrt{\ln 2}}\right)^{2}}\right] & x \geqslant x_{\mathrm{a}} \\
& x \geqslant x_{\mathrm{a}} .\end{cases}
\end{aligned}
$$

Fitting this expression to the suppression factor of Badnell et al. (1993) for C IV (which was computed at $T=10^{5} \mathrm{~K}$ ) yielded the values $\mu=0.372, w=4.969$, and $x_{\mathrm{a}}=0.608$, and this parameterization formula is found to be accurate to within $5 \%$ for all densities considered (see Figure 1).

\subsection{Generalized High-temperature Suppression Formula}

Given the suppression formula for Li-like C IV, corresponding to ionic charge $q_{0}=3$ and temperature $T_{0}=10^{5} \mathrm{~K}$, we wish to generalize this expression to other Li-like ions of charge $q$ and (high) $T$ according to the following qualitative guidelines. It is well known that density effects scale as $q^{7}$ - see Bates et al. (1962) and Burgess \& Summers (1969). The activation density is attained when the reionization rate in Equation (3), which depends linearly on the density, becomes comparable to the radiative stabilization rate in Equation (2). The radiative rate is independent of density and temperature, but scales with charge as $A_{r} \sim q^{4}$, whereas the electron-impact ionization rate depends on all three, viz., $n_{\mathrm{e}} \alpha_{\mathrm{eII}} \sim n_{\mathrm{e}} q^{-3} T^{-1 / 2}$. An initial suggestion is that the activation density is attained when these two are approximately equal, i.e.,

$$
n_{\mathrm{e}, \mathrm{a}} q^{-3} T^{-1 / 2} \sim q^{4},
$$

indicating that the activation density should scale as $n_{\mathrm{e}, \mathrm{a}} \sim$ $q^{7} T^{1 / 2}$, if the above qualitative discussion holds. The $\log$ activation density for all $q$ and $T$ might therefore be expected to obey the scaling relationship

$$
x_{\mathrm{a}}(q, T)=x_{\mathrm{a}}\left(q_{0}, T_{0}\right)+\log _{10}\left[\left(\frac{q}{q_{0}}\right)^{7}\left(\frac{T}{T_{0}}\right)^{1 / 2}\right],
$$

where $x_{\mathrm{a}}\left(q_{0}, T_{0}\right)=0.608, q_{0}=3$, and $T_{0}=10^{5} \mathrm{~K}$ are the (log) activation density, the charge, and the temperature for the C IV case treated by Badnell et al. (1993). We note that this expression, when applied to Li-like $\mathrm{O}$ VI, gives an increase in the activation density by a factor of $(5 / 3)^{7}=35.7$, in agreement with the approximate factor of 40 found by Davidson (1975). Furthermore, when scaled in temperature, the formula gives fairly good agreement with the suppression results of Davidson (1975) for C IV at $T=1.5 \times 10^{4} \mathrm{~K}$ (see Figure 1$)$. 


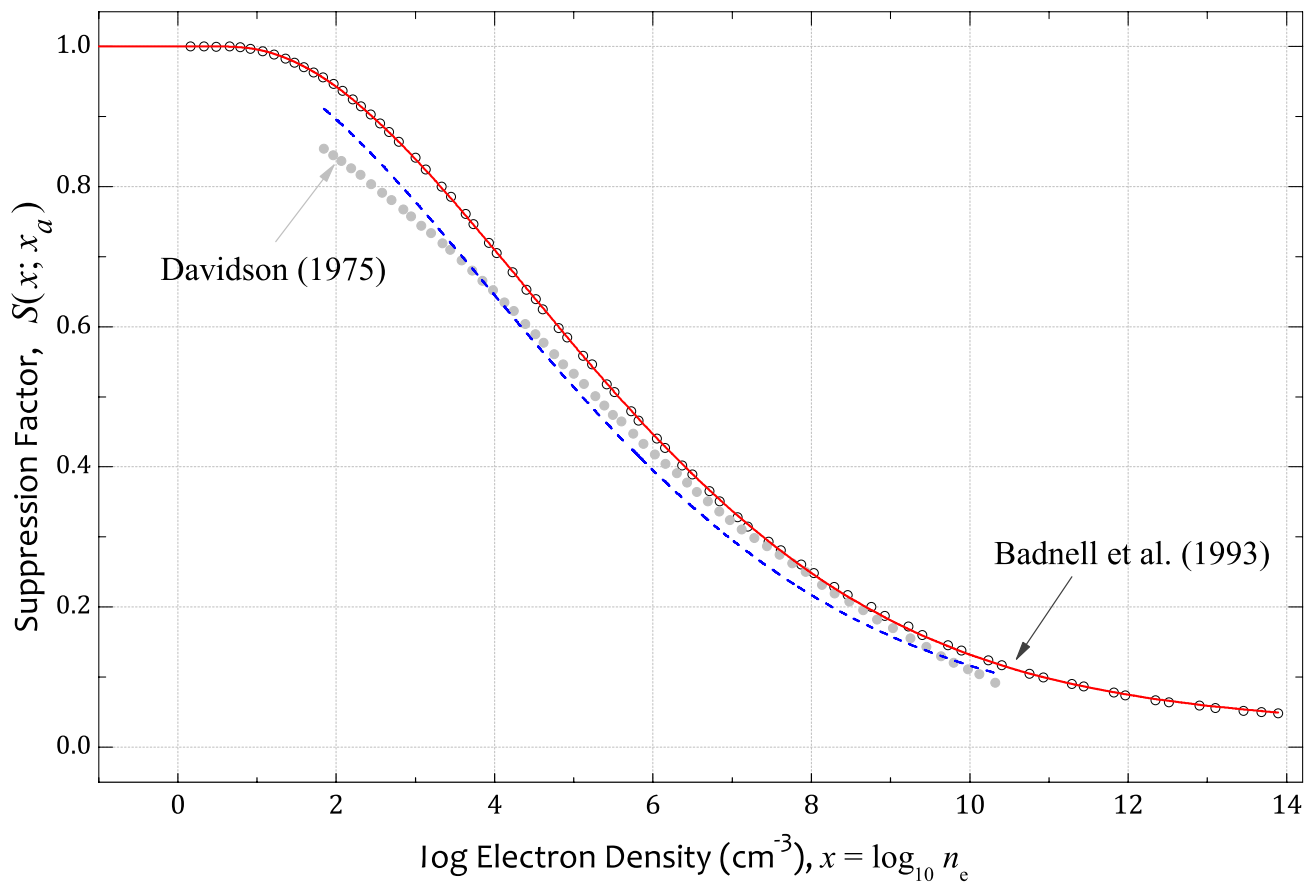

Figure 1. Pseudo-Voigt fit of the suppression factor for C IV, as given in Equation (5) with a scaled activation density as given by Equation (7), shown for two different temperatures. The red solid curve shows that the parameterization for $T=1 \times 10^{5} \mathrm{~K}$, corresponding to an activation density of $x_{\mathrm{a}}=0.608$ (with $\mu=0.372$ and $w=4.969$ ), is in close agreement with the actual data of Badnell et al. (1993; open circles). The blue dashed curve is the parameterization for $T=1.5 \times 10^{4} \mathrm{~K}$, using instead an activation density of $x_{\mathrm{a}}=0.196$ (and the same $\mu$ and $w$ ), and giving satisfactory agreement with the data of Davidson (1975; solid circles).

(A color version of this figure is available in the online journal.)

\subsubsection{Fit to the Collisional Radiative Data}

The preceding treatment reasonably extends the CIV suppression factor at $10^{5} \mathrm{~K}$ to other high temperatures and to other Li-like ions. However, we need suppression factors applicable to all ionization stages of all elements up to at least $\mathrm{Zn}$ for a general implementation within Cloudy. Unfortunately, detailed collisional-radiative modeling data with state-of-the-art DR data are still rather limited. However, extensive tables of effective recombination rate coefficients have been computed by Summers (1974 \& 1979) for a wide variety of isoelectronic sequences, charge states, temperatures, and densities. The treatment of DR there is somewhat simplified, but we only require the ratio of finite- to zero-density rate coefficients to determine the suppression factor. We then combine this ratio with our state-of-the-art zero density DR rate coefficients again for use within Cloudy. This ratio is much less sensitive to the specific treatment of DR.

The rather simplistic scaling formula in Equation (7) was found to be inadequate when applied to the extensive tabulation of suppression factors found in Summers (1974 \& 1979). Instead, in order to fit the data accurately, a more generalized formula was arrived at, where a pseudo-Gaussian, corresponding to $\mu=0$ in Equation (5), was more appropriate,

$$
S^{N}(x ; q, T)=\left\{\begin{array}{cl}
1 & x \leqslant x_{a}(q, T, N) \\
e^{-\left(\frac{x-x_{a}(q, T, N)}{w / \sqrt{\ln 2}}\right)^{2}} & x \geqslant x_{a}(q, T, N)
\end{array} .\right.
$$

Furthermore, the activation density was found to be best represented by the function

$$
x_{a}(q, T, N)=x_{a}^{0}+\log _{10}\left[\left(\frac{q}{q_{0}(q, N)}\right)^{7}\left(\frac{T}{T_{0}(q, N)}\right)^{1 / 2}\right],
$$

where the variables $q_{0}(q, N)$ and $T_{0}(q, N)$ are taken to be functions of the charge $q$ and the isoelectronic sequence, labeled by $N$. A fit of the suppression factors of Summers (1974 \& 1979) for all ions yielded a global $(\log )$ activation density $x_{a}^{0}=10.1821$, $w=5.64548$, and more complicated expressions for the zeropoint temperature $T_{0}$ and charge $q_{0}$. These were found to depend on both the ionic charge $q$ and the isoelectronic sequence $N$, viz.,

$$
T_{0}(q, N)=5 \times 10^{4}\left[q_{0}(q, N)\right]^{2}
$$

and

$$
q_{0}(q, N)=(1-\sqrt{2 / 3 q}) A(N) / \sqrt{q}
$$

where

$$
A(N)=12+10 N_{1}+\frac{10 N_{1}-2 N_{2}}{N_{1}-N_{2}}\left(N-N_{1}\right)
$$

depends on the isoelectronic sequence in the periodic table according to the specification of the parameters

$$
\begin{aligned}
& \left(N_{1}, N_{2}\right) \\
& \quad=\left(\begin{array}{llll}
(3,10) & N \in 2 \text { nd row } & (37,54) & N \in 5 \text { th row } \\
(11,18) & N \in \text { 3rd row } & (55,86) & N \in 6 \text { th row } \\
(19,36) & N \in 4 \text { th row } & (87,118) & N \in 7 \text { th row }
\end{array}\right) .
\end{aligned}
$$

However, even this rather complicated parameterization was inadequate for the lower isoelectronic sequences $N \leqslant 5$, and for these we explicitly list the optimal values for $A(N)$ in Table 1. Furthermore, at electron temperatures and/or ionic charges for which the $q$-scaled temperature $\theta \equiv T / q^{2}$ was very low $\left(\theta \leqslant 2.5 \times 10^{4} \mathrm{~K}\right)$, a further modification to the coefficients 

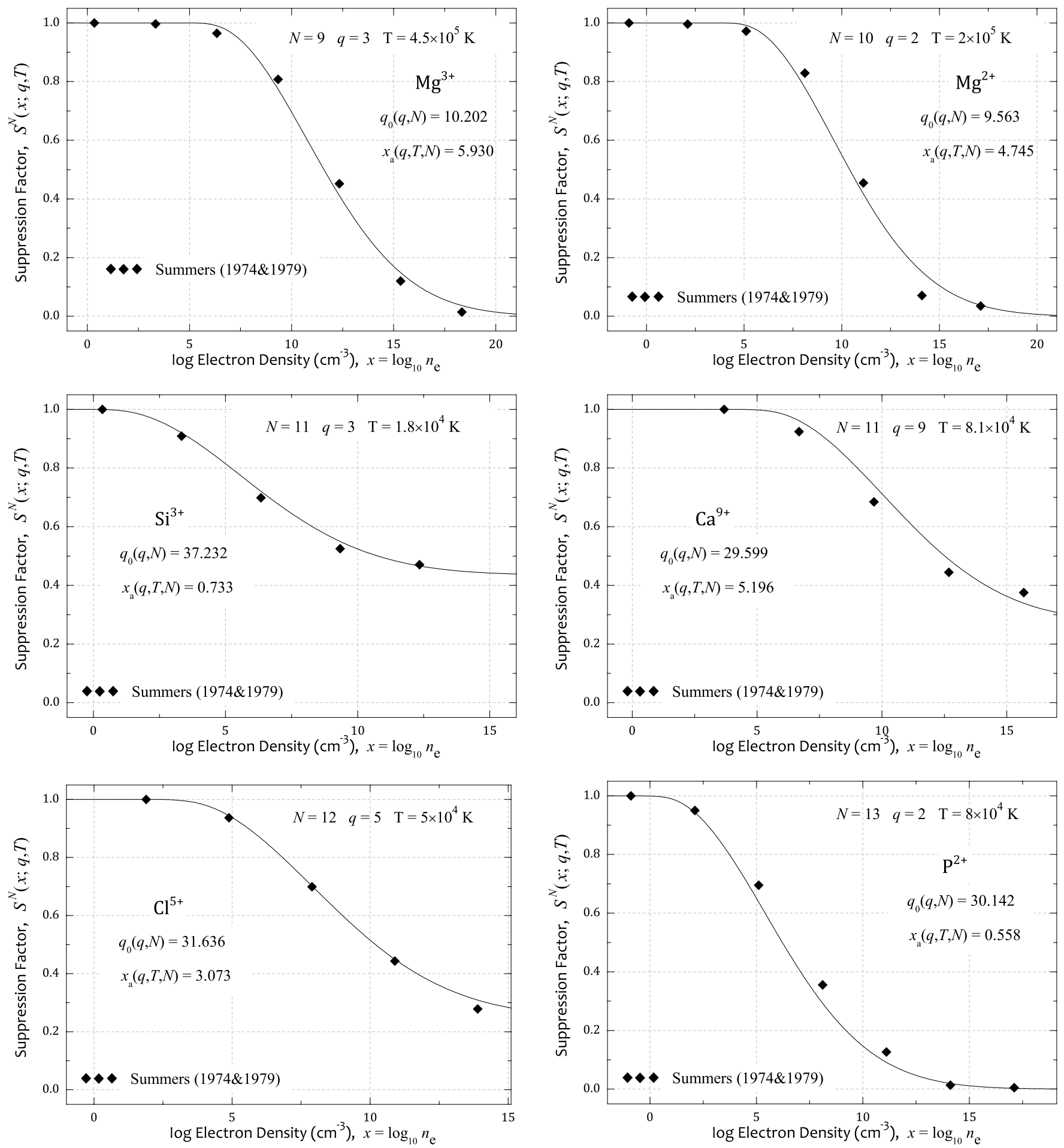

Figure 2. A comparison between the present parameterized suppression factor and the collisional radiative results of Summers (1974 \& 1979) for a sample of ions and temperatures, as a function of density.

$A(N)$ for $N \leqslant 5$ is necessary in that the values in Table 1 should be multiplied by a factor of two.

The final formulation for the suppression curve (Equation (8)), and its rather complicated activation density (Equations (9)-(13)), has been found to model the entire database of ions, temperatures, and densities considered in the Summers (1974 \& 1979) data fairly well. To illustrate the general level of agreement over a large range of ions and environments, we compare our parameterized model formulation to the actual suppression data from that report (Summers 1974 \& 1979) for a few selected cases in Figure 2. In order to quantify more fully the extent of agreement, we focus on the case of iron ions, for which we study density effects on ionization balance determination in the next section. A comparison is shown in Figure 3 between our predicted suppression factors and the data from the Summers (1974 \& 1979) report. It is seen that our model fits those data to within $21 \%$ for all densities, temperatures, and ionic stages reported (Summers 1974 \& 1979). More 

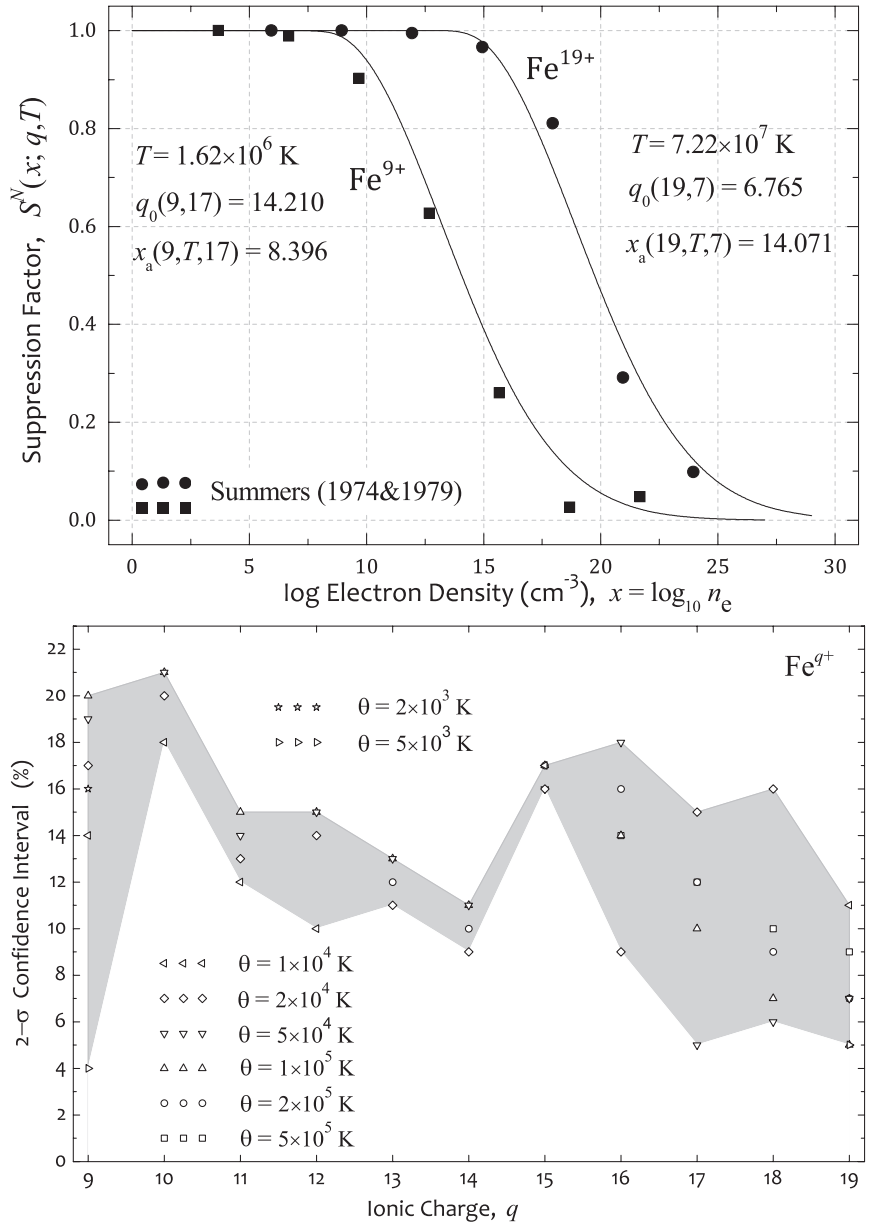

Figure 3. Agreement between the suppression curve of Equation (8) and the Summers (1974 \& 1979) data for all iron ions $\mathrm{Fe}^{q+}, q=9-19$. The upper panel shows the detailed level of agreement of the two end cases, $\mathrm{Fe}^{9+}$ and $\mathrm{Fe}^{19+}$. The lower panel shows the $2 \sigma(95.4 \%)$ confidence level as a function of charge state; this means that $95.4 \%$ of all density data points in the Summers (1974 \& 1979) data, for the given charge and temperature, are within that percentage of the prediction from Equation (8). The symbols denote different values of the scaled temperature $\theta=T / q^{2}$.

Table 1

Modified $A(N)$ Coefficients from Equation (12)

\begin{tabular}{lcc}
\hline \hline Sequence & $N$ & $A(N)^{\mathrm{a}}$ \\
\hline H-like & 1 & 16 \\
He-like & 2 & 18 \\
Li-like & 3 & 66 \\
Be-like & 4 & 66 \\
B-like & 5 & 52 \\
\hline
\end{tabular}

Note. ${ }^{a}$ These must be multiplied by 2.0 if $\theta \leqslant$ $2.5 \times 10^{4} \mathrm{~K}$.

broadly, we have applied a similar $2 \sigma$ analysis to all ions in that report, at all temperatures and densities, and find the same agreement (20\%-26\% confidence level).

Lastly, it is of interest to investigate how our final suppression factor in Equation (8) compares to our original, motivating, formulation of Equation (8) for C IV, shown in Figure 4. There is generally good qualitative agreement. However, it is seen that the original formulation, based on the Badnell et al. (1993) results, shows a somewhat stronger suppression effect up to $x \approx 11$. This is likely due to the more accurate treatment of the partial DR data of Badnell et al. (1993) entering the collisional-radiative modeling, although some difference due to the collisional-radiative modeling itself may also be present. This indicates that even collisional plasmas require collisionalradiative modeling with state-of-the-art DR data. The stronger suppression tails off at $x \gtrsim 11$ as three-body recombination starts to become relevant and, at even higher densities (not shown), causes the suppression factor to rise (since it is a ratio of effective recombination rate coefficients, i.e., includes threebody recombination).

\subsection{Suppression Formula at Low Temperatures}

The preceding formulation was based on the suppression factor found by Summers (1974 \& 1979) for electron collisionally ionized plasmas, i.e., at higher temperatures, where DR is dominated by high- $n$ resonances attached to a dipole-allowed core excited state. In photoionization equilibrium, however, the temperature at which a given ion forms is substantially smaller than that found in the electron collisional case. Due to the lower kinetic temperatures, DR occurs mainly through energetically low-lying autoionizing states, often via non-dipole coreexcitations for which radiative stabilization is by the (outer) Rydberg electron. These states are not, in general, as susceptible to density suppression as their high- $n$ counterparts, and so it may be necessary to modify the preceding suppression formulation.

We first consider sequences with partially-occupied $p$-subshells in the ground state, which include the B-like $2 p\left({ }^{2} P_{1 / 2,3 / 2}\right)$, C-like $2 p^{2}\left({ }^{3} P_{0,1,2}\right)$, O-like $2 p^{4}\left({ }^{3} P_{0,1,2}\right)$, F-like $2 p^{5}\left({ }^{2} P_{3 / 2,1 / 2}\right), \quad$ Al-like $3 p\left({ }^{2} P_{1 / 2,3 / 2}\right)$, Si-like $3 p^{2}\left({ }^{3} P_{0,1,2}\right)$, S-like $3 p^{4}\left({ }^{3} P_{0,1,2}\right)$, and Cl-like $3 p^{5}\left({ }^{2} P_{3 / 2,1 / 2}\right)$ systems. For these sequences, there is fine-structure splitting in the ground state and a correspondingly small excitation energy, $\epsilon_{N}$, giving dielectronic capture into high principal quantum numbers (because of the Rydberg relation $\left.q^{2} / n^{2} \leqslant \epsilon_{N}\right)$. Stabilization is via $n \rightarrow n^{\prime}$ transitions and the recombined final state is built upon an excited parent. Ultimately, it is the strength of collisional coupling of this final state with the continuum which determines whether recombination or ionization prevails. As the density increases, collisional LTE extends further down the energy spectrum. It is difficult to give a general statement about the position of such final states relative to the ionization limit. So, we assume a worst case scenario, i.e., that such states are subject to suppression, and we use the preceding suppression formula. If density effects are found to be small in photoionized plasmas then this is likely sufficient. If they appear to be significant then a more detailed treatment based on collisional-radiative modeling will be needed. Thus, for these systems, we retain the same suppression formula developed above (Equations (8)-(13)), viz. $S^{N}(x, q, T)$, for $N=\{5,6,8,9,13,14,16,17\}$, and for all $q$ and $T$.

For the hydrogenic and the closed-shell He-like and Ne-like cases, on the other hand, the excitations proceed via an increase in core principal quantum number $-1 s \rightarrow 2 s$ or $\{2 s, 2 p\} \rightarrow\{3 s, 3 p, 3 d\}$ - giving the dominant dielectronic capture into the low- $n<10$ resonances. Even following core radiative stabilization, these low-lying states are impervious to collisional reionization for the range of densities $x \leqslant 10$, and thus we set $S^{N}(x, q, T)=1.0$ for $N=\{1,2,10\}$. However, at densities $x>10$, the Summers (1974 \& 1979) data for these three isoelectronic sequence show suppression factors that are fit well by the usual Equation (8), so we do not modify $S^{N}(x, q, T)$ for these cases. 


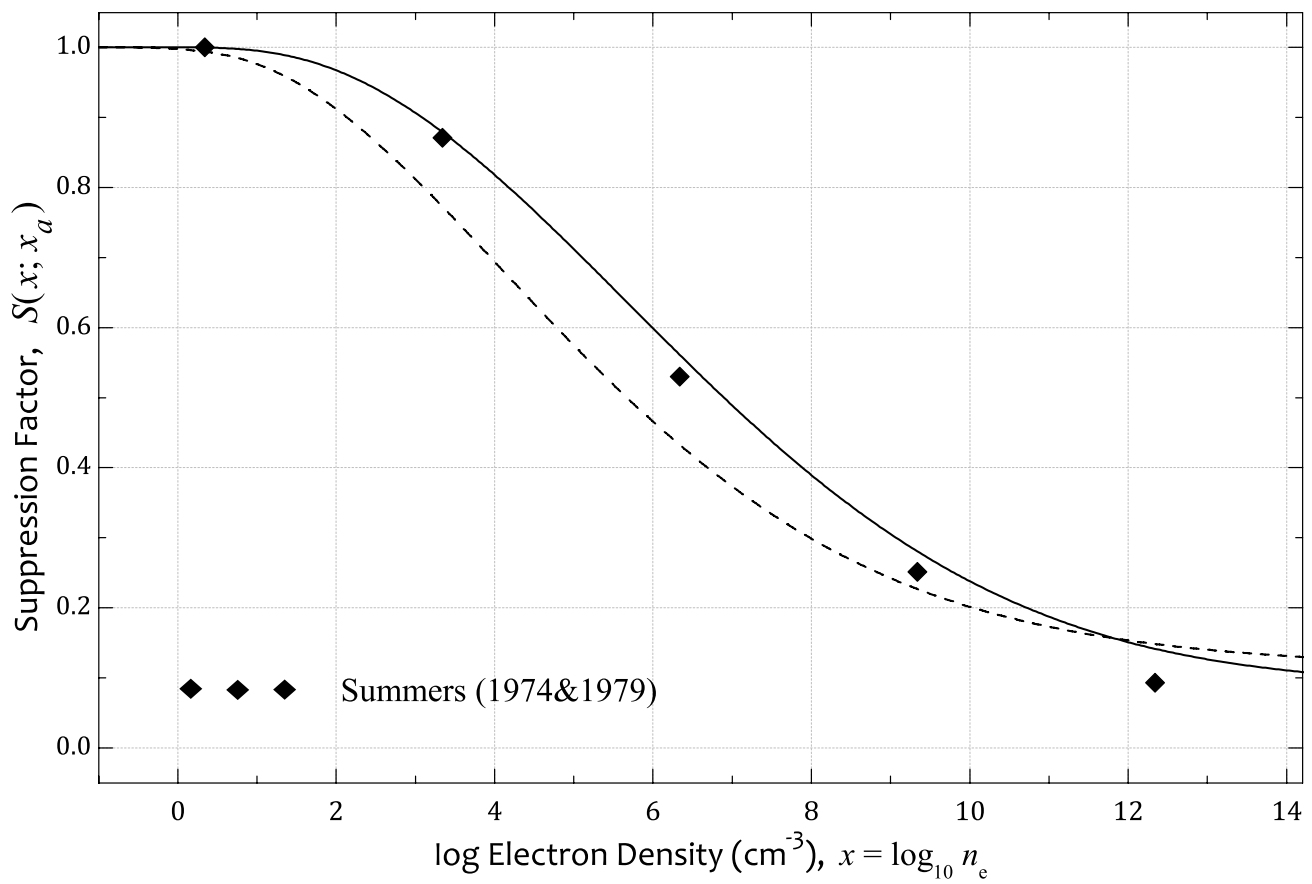

Figure 4. A comparison between the final suppression factor of Equation (8) (solid line), corresponding to a pseudo-Gaussian profile with activation density $x_{\mathrm{a}}=0.8314\left(q_{0}=40.284\right)$ and width $w=5.64548$, the Summers (1974 \& 1979) data points (solid diamonds), and the original formulation of Equation (5) (dashed line), corresponding to a pseudo-Voigt profile with activation density $x_{\mathrm{a}}=0.608$, width $w=4.696$, and mixture coefficient $\mu=0.372$. The temperature $T=1 \times 10^{5} \mathrm{~K}$ is the same as in Figure 1.

Lastly, we consider the intermediate isoelectronic sequences for which excitation arises from neither a fine-structure splitting of the ground state nor a change in principal quantum number of the core. These include the Li-like $2 s \rightarrow 2 p$, Be-like $2 s^{2} \rightarrow$ $2 s 2 p$, N-like $2 s^{2} 2 p^{3}\left({ }^{4} S\right) \rightarrow 2 s 2 p^{4}\left({ }^{4} P\right)$, Na-like $3 s \rightarrow 3 p$, Mg-like $3 s^{2} \rightarrow 3 s 3 p$, and P-like $3 s^{2} 3 p^{3}\left({ }^{4} S\right) \rightarrow 3 s 3 p^{4}\left({ }^{4} P\right)$ cases up through the third row sequences. Any large lowtemperature DR contribution arising from near threshold resonances is to low-lying states, for which suppression is negligible, i.e., the high-temperature suppression factor must be switched off $\left(S^{N} \rightarrow 1\right)$ at low $T$.

To illustrate the general demarcation between low- $T$ and high$T$ DR, we first consider DR of CIV, an overview of which is depicted in Figure 5. The DR cross section, shown in the inset, is dominated by two features. The first is the $n \rightarrow \infty$ accumulation of resonances at the $\epsilon=8 \mathrm{eV}$ series limit-those which can be treated in the usual high- $T$ fashion (Burgess 1965; Burgess \& Summers 1969) and are therefore susceptible to suppression according to our formulation above. However, there is a second strong contribution from the lowest accessible resonances just above the threshold electron energy, which, according to the Rydberg consideration $3^{2} / n^{2} \approx \epsilon_{3}=0.6 \mathrm{Ryd}$, occur here for $n=4$. More generally, these low-lying states are typical of the low-lying DR spectrum (Nussbaumer \& Storey 1984). ${ }^{6}$ The $1 s^{2} 2 p 4 l$ resonances decay predominantly to the $1 s^{2} 2 s 2 p$, $1 s^{2} 2 p^{2}$ and $1 s^{2} 2 s 4 l$ states. These states lie well below the ionization limit and so are not susceptible to further reionization. Since there should be no density suppression then, we seek a modified suppression factor which tends toward unity (i.e., no suppression) at lower temperatures.

In order to make a smooth transition from the high- $T$ suppression factor $S^{N}(x ; q, T)$ given in Equation (8), which

\footnotetext{
6 We note that the CIV $n=4$ resonance manifold has been the subject of
} further near-threshold density-dependent effects (Pindzola et al. 2011).

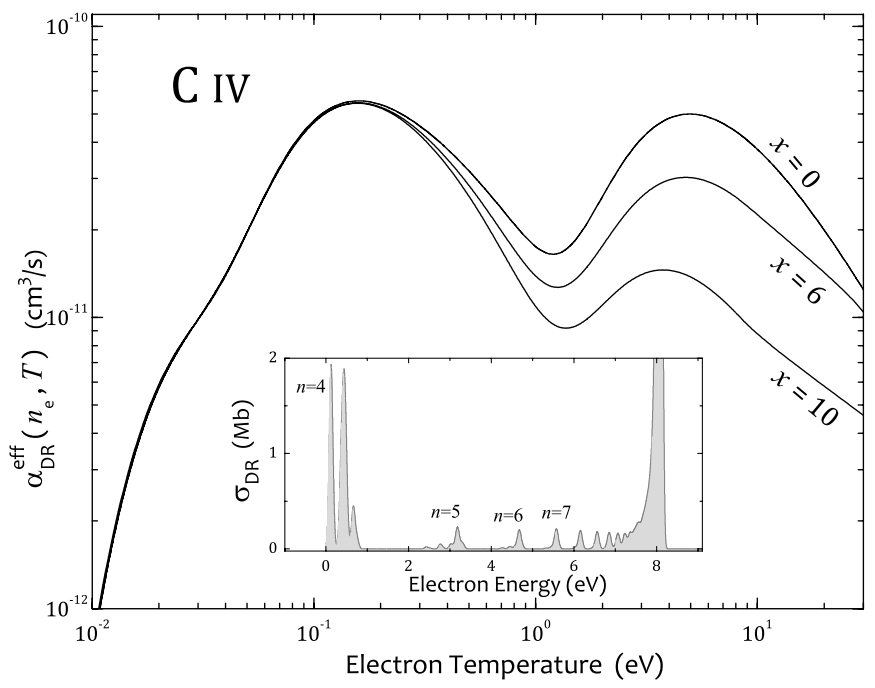

Figure 5. DR of CIv. The inset shows the (zero-density) DR cross section convoluted with a $0.1 \mathrm{eV}$ FWHM Gaussian. The spectrum is dominated by two features: the $n=4$ DR resonance manifold below $1.0 \mathrm{eV}$ and the $n \rightarrow \infty$ Rydberg resonances accumulating at the $2 s \rightarrow 2 p$ series limit $\epsilon_{3}\left(q_{0}\right) \approx 8 \mathrm{eV}$. The main figure shows the effective DR rate coefficient for several densities. Our modified suppression formulation for $x>0$, using Equations (8) and (14), ensures that the high- $T$ peak, corresponding to the $n \rightarrow \infty$ Rydberg series of resonances, is suppressed but the low- $T$ peak, corresponding to the $n=4$ resonances, is not suppressed.

is appropriate for the high- $T$ peak region $k T \approx k T_{\max }=2 \epsilon_{N} / 3$, to the low- $T$ region, where $S^{N} \rightarrow 1$, we use the modified factor

$$
S^{N}(x ; q, T) \rightarrow 1-\left[1-S^{N}(x ; q, T)\right] \exp \left(-\frac{\epsilon_{N}(q)}{10 k T}\right),
$$




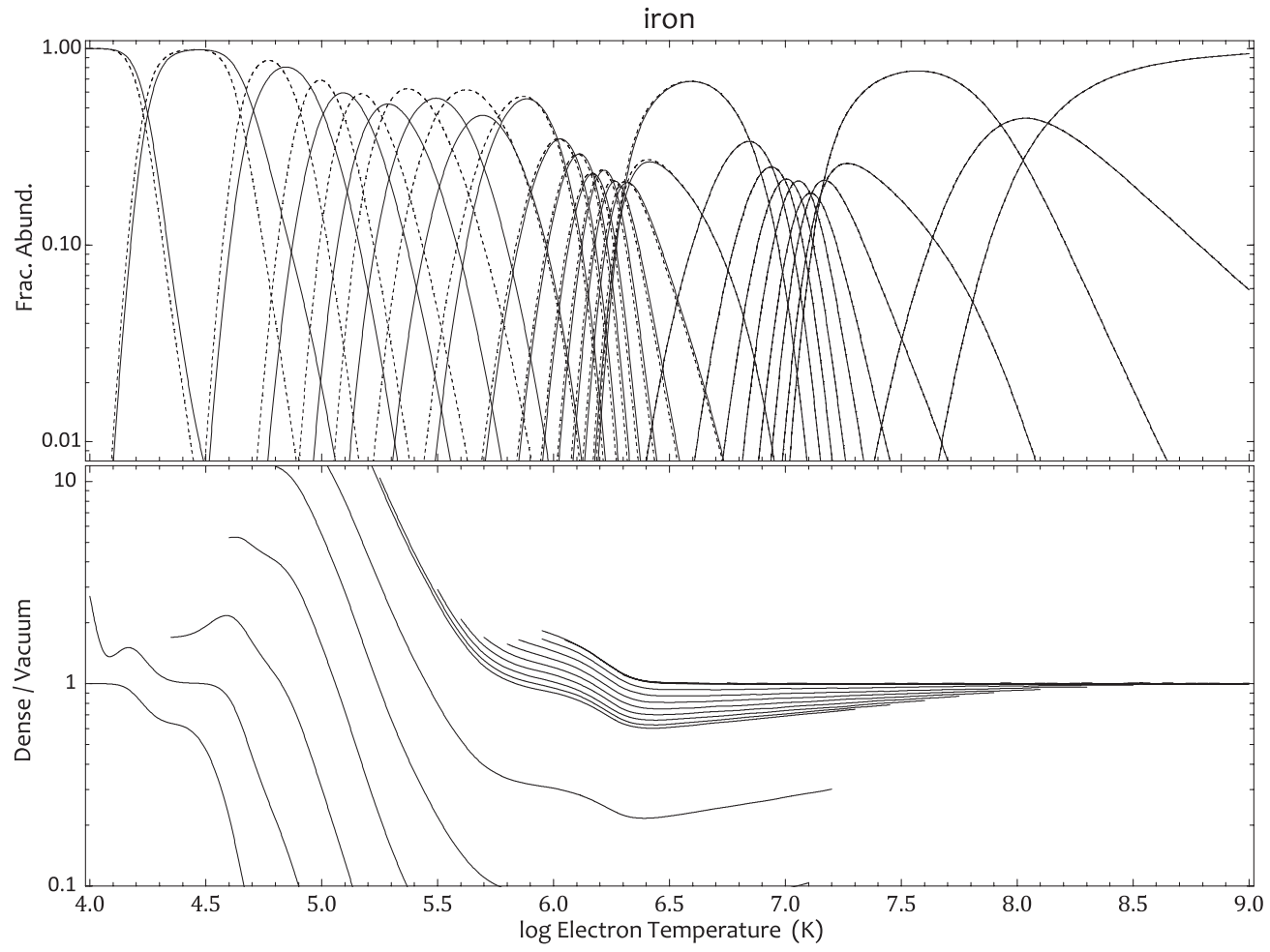

Figure 6. Upper panel: collisional ionization fractional abundance vs. electron temperature for all ionization stages of Fe. The solid curves correspond to a density of $1 \mathrm{~cm}^{-3}$ and the dashed curves correspond to a density of $10^{10} \mathrm{~cm}^{-3}$. From left to right, the curves range from Fe I to Fe xxvII. Lower panel: ratio of the calculated fractional abundances for the two densities.

where $\epsilon_{N}(q)=8 \mathrm{eV}$ for the particular case of $\mathrm{C} \mathrm{IV}(N=3$ and $q=3$ ). As seen in Figure 5, the density-dependent effective DR rate coefficient, $\alpha_{D R}^{\text {eff }}\left(n_{e}, T\right)$, indeed satisfies the requirement that the high- $T$ peak is suppressed according to the formulation of Badnell et al. (1993) whereas suppression is totally turned off for the lower- $T$ peak.

We have investigated the application of Equation (14) for all ions that exhibit these same low- $T$ resonance features, namely, all isoelectronic sequences $N=\{3,4,7,11,12,15\}$, and we have found that the correct transitioning from suppression at the high- $T$ peak to no suppression at low- $T$ is indeed satisfied, provided, of course, that the appropriate dipoleallowed excitation energy $\epsilon_{N}(q)$ is employed. For efficient representation, the excitation energies along each isoelectronic sequence are parameterized by the expression

$$
\epsilon_{N}(q)=\sum_{j=0}^{5} p_{N, j}\left(\frac{q}{10}\right)^{j}
$$

These parameters, which are determined by fitting the above expression to the available NIST excitation energies (Ralchenko et al. 2011), are listed in Table 2.

We note that all isoelectronic sequences and ionization stages are now included in this prescription-our final comprehensive model for treating DR suppression, albeit in a simplified fashion. For those ions with fine-structure splitting in the ground state, we have $\epsilon_{N}(q) \approx 0$. (We apply this generally also for $\operatorname{Ar}-$ like sequences and above $(N \geqslant 18)$, based on the density of states-see, for example, Badnell (2006b).) For the closedshell cases, on the other hand, we have $\epsilon_{N}(q) \rightarrow \infty$. Thus, $S^{N}(x ; q, T)=1$ for hydrogenic and closed-shell systems, i.e., there is no suppression (for $x \leqslant 10$ ). Lastly, for the intermediate cases, the suppression factor is gradually increased toward unity at lower temperatures and begins to admit low- $n$ DR resonances.

\section{RESULTS}

The suppression factors derived here have been applied to the state-of-the-art total DR rate coefficients taken from the most recent DR database. ${ }^{7}$ These modified data have been incorporated into version $\mathrm{C} 13$ of the plasma simulation code Cloudy, most recently described by Ferland et al. (2013). Cloudy can do simulations of both photoionized and collisionally ionized cases, and we show the effects of collisional suppression on both.

Figure 6 shows the ionization distribution of iron for the collisional ionization case. Figure 7 shows a similar calculation for photoionization equilibrium. Both show two hydrogen densities, $1 \mathrm{~cm}^{-3}$, where collisional suppression of DR should be negligible, and $10^{10} \mathrm{~cm}^{-3}$, where collisional suppression should greatly affect the rates for lower charges and temperatures. The upper panel shows the ionization fractions themselves, for these two densities, while the lower panel shows the ratio of the high to low density abundances.

Cloudy's assumptions in computing collisional ionization equilibrium, as shown in Figure 6, have been described by Lykins et al. (2013). It is determined by the balance between collisional ionization from the ground state and recombination by radiative, dielectronic, and three body recombination to all levels of the recombined species.

The photoionization case shown in Figure 7 depicts the active galactic nucleus spectral energy distribution (SED), described by Mathews \& Ferland (1987), as a function of the ionization

\footnotetext{
7 http://amdpp.phys.strath.ac.uk/tamoc/DATA/
} 


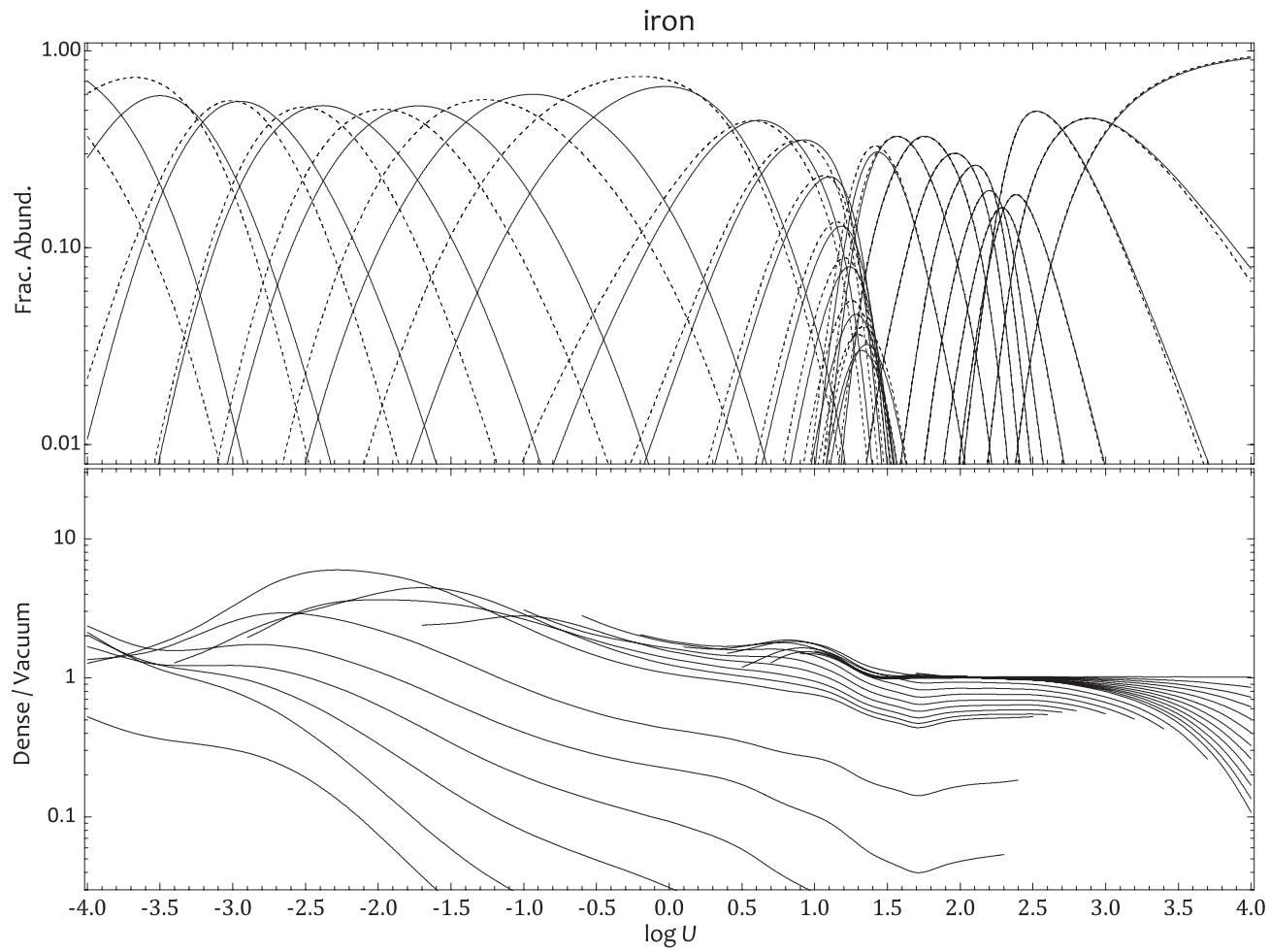

Figure 7. Upper panel: photoionization fractional abundance vs. the ionization parameter $U$ for all ionization stages of Fe. The solid curves correspond to a density of $1 \mathrm{~cm}^{-3}$ and the dashed curves correspond to a density of $10^{10} \mathrm{~cm}^{-3}$. From left to right, the curves range from Fe I to Fe XxvII. Lower panel: ratio of the calculated fractional abundances for the two densities.

Table 2

Fitting Coefficients for the Excitation Energies $\epsilon_{N}(q)=\sum_{j=0}^{5} p_{N, j}(q / 10)^{j}$, in eV

\begin{tabular}{|c|c|c|c|c|c|c|c|}
\hline Sequence & $N$ & $p_{N, 0}$ & $p_{N, 1}$ & $p_{N, 2}$ & $p_{N, 3}$ & $p_{N, 4}$ & $p_{N, 5}$ \\
\hline Li-like & 3 & $1.963[+0]$ & $2.030[+1]$ & $-9.710[-1]$ & $8.545[-1]$ & $1.355[-1]$ & $2.401[-2]$ \\
\hline Be-like & 4 & $5.789[+0]$ & $3.408[+1]$ & $1.517[+0]$ & $-1.212[+0]$ & $7.756[-1]$ & $-4.100[-3]$ \\
\hline N-like & 7 & $1.137[+1]$ & $3.622[+1]$ & $7.084[+0]$ & $-5.168[+0]$ & $2.451[+0]$ & $-1.696[-1]$ \\
\hline Na-like & 11 & $2.248[+0]$ & $2.228[+1]$ & $-1.123[+0]$ & $9.027[-1]$ & $-3.860[-2]$ & $1.468[-2]$ \\
\hline Mg-like & 12 & $2.745[+0]$ & $1.919[+1]$ & $-5.432[-1]$ & $7.868[-1]$ & $-4.249[-2]$ & $1.357[-2]$ \\
\hline P-like & 15 & $1.428[+0]$ & $3.908[+0]$ & $7.312[-1]$ & $-1.914[+0]$ & $1.051[+0]$ & $-8.992[-2]$ \\
\hline H-, He-, Ne-like & $1,2,10$ & $\infty^{\mathrm{a}}$ & 0.0 & 0.0 & 0.0 & 0.0 & 0.0 \\
\hline B-, C-, O-, F-like & $5,6,8,9$ & 0.0 & 0.0 & 0.0 & 0.0 & 0.0 & 0.0 \\
\hline \multirow[t]{2}{*}{ Al-, Si-, S-, Cl-like } & $13,14,16,17$ & 0.0 & 0.0 & 0.0 & 0.0 & 0.0 & 0.0 \\
\hline & $\geqslant 18$ & 0.0 & 0.0 & 0.0 & 0.0 & 0.0 & 0.0 \\
\hline
\end{tabular}

Notes. Numbers in square brackets denote powers of 10 .

${ }^{\text {a }}$ Reset to 0.0 for $x>10$.

parameter

$$
U \equiv \frac{\Phi_{H}}{n_{H} c},
$$

where $\Phi_{H}$ is the hydrogen-ionizing photon flux, $n_{H}$ is the density of hydrogen, and $c$ is the speed of light. There is only an indirect relationship between the gas kinetic temperature and the ionization of the gas in this case. Here, the level of ionization is determined by a balance between photoionization by the energetic continuum and the total recombination rate.

The lower panels of Figures 6 and 7 show that the amount that the ionization increases due to DR suppression can be large - the ratio can easily exceed 1 dex. Clearly, these results demonstrate that density effects on the ionization balance need to be considered more precisely.

\section{CONCLUSION}

We have investigated the effects of finite densities on the effective DR rate coefficients by developing a suppression factor model, which was motivated by the early work of Badnell et al. (1993) for C IV and extended to all other ions using physicallymotivated scaling considerations, and more precise fitting of collisional-radiative data (Summers 1974 \& 1979). Accurate zero-density DR rate coefficients were then multiplied by this suppression factor and introduced into Cloudy to study the finite-density effects on computed ionization balances of both collisionally ionized and photoionized plasmas. It is found that the difference in ionization balance between the near-zero and finite-density cases is substantial, and thus there is sufficient justification for further studies of collisional suppression from generalized collisional-radiative calculations. This is expected 
to impact the predictions of the ionization balance in denser cosmic gases such as those found in nova and supernova shells, accretion disks, and the broad emission line regions in active galactic nuclei.

The present results are intended to be preliminary, and to demonstrate the importance of density effects on DR in astrophysical plasmas. Given the approximations adopted, we suggest that their incorporation into models (e.g., via Cloudy) be used with a little caution. For example, one might run models with and without the effects of suppression at finite density, especially in modeling higher density plasmas (e.g., the broad emission line region in quasars). Nevertheless, it is nearly half a century since Burgess \& Summers (1969) demonstrated significant density effects on DR, and it is time that some representation exists within astrophysical modeling codes to assess its impact on the much more rigorous demands made by modern day modeling, especially given its routine incorporation by magnetic fusion plasma modeling codes. In the longer term, we intend to present results based on detailed collisionalradiative calculations using state-of-the-art state-specific DR rate coefficients.

D.N., T.W.G., and K.T.K. acknowledge support by NASA (NNX11AF32G). G.J.F. acknowledges support by NSF (1108928; and 1109061), NASA (10-ATP10-0053, 10-ADAP10-0073, and NNX12AH73G), and STScI (HST-AR12125.01, GO-12560, and HST-GO-12309). UK undergraduates Mitchell Martin and Terry Yun assisted in coding the DR routines used here. N.R.B. acknowledges support by STFC (ST/J000892/1).

\section{REFERENCES}

Badnell, N. R. 2006a, ApJS, 167, 334

Badnell, N. R. 2006b, ApJL, 651, L73

Badnell, N. R., O’Mullane, M. G., Summers, H. P., et al. 2003, A\&A, 406, 1151

Badnell, N. R., Pindzola, M. S., Dickson, W. J., et al. 1993, ApJL, 407, L91

Bates, D. R., Kingston, A. E., \& McWhirter, R. W. P. 1962, RSPSA, 267, 297

Burgess, A. 1965, ApJ, 141, 1588

Burgess, A., \& Summers, H. P. 1969, ApJ, 157, 1007

Davidson, K. 1975, ApJ, 195, 285

Ferland, G. J., Porter, R. L., van Hoof, P. A. M., et al. 2013, RMxAA, 49, 137

Jordan, C. 1969, MNRAS, 142, 501

Lykins, M., Ferland, G. J., Porter, R. L., et al. 2013, MNRAS, 429, 3133

Mathews, W. G., \& Ferland, G. J. 1987, ApJ, 323, 456

Nussbaumer, H., \& Storey, P. J. 1984, A\&AS, 56, 293

Osterbrock, D. E., \& Ferland, G. J. 2006, Astrophysics of Gaseous Nebulae and Active Galactic Nuclei (2nd ed.; Sausalito, CA: University Science Books)

Pindzola, M. S., Loch, S. D., \& Robicheaux, F. 2011, PhRvA, 83, 042705

Ralchenko, Y., Kramida, A. E., Reader, J., \& NIST ASD Team 2011, National Institute of Standards and Technology, http://physics.nist.gov/asd

Summers, H. P. 1972, MNRAS, 158, 255

Summers, H. P. 1974, MNRAS, 169, 633

Summers, H. P. 1974 \& 1979, Appleton Laboratory Internal Memorandum IM367 \& Re-issued with Improvements as AL-R-5

Summers, H. P., \& Hooper, M. B. 1983, PIPh, 25, 1311

Verner, D. A., \& Yakovlev, D. G. 1995, A\&AS, 109, 125

Voronov, G. S. 1997, ADNDT, 65, 1

Wertheim, G. K., Butler, M. A., West, K. W., \& Buchanan, D. N. E. 1974, RScI, 45,1369 